\title{
BLAST: BLOCKCHAIN ALGORITHM FOR SECURE TRANSACTION
}

\author{
Rahul Johari ${ }^{1}$ and Anurag Singh Parihar ${ }^{2}$ \\ ${ }^{1}$ SWINGER: Security, Wireless, IoT Network of group of Engineering and \\ Research Lab, University School of Information, Communication Technology \\ (USICT), Guru Gobind Singh Indraprastha University, Sector-16C, Dwarka, New \\ Delhi, India \\ ${ }^{1}$ swinger@ipu.ac.in, ${ }^{2} 1988$. anurag@gmail.com
}

\begin{abstract}
Cryptography and security are important areas in industry to store large voluminous, useful and private data. With the evolution of business to business 4.0, per-forming secure commercial transactions on the web have become important. In business 4.0, emerging technologies such as block chain, cloud computing, et. al. are built on the concepts of cryptography and security. The need for the movement towards business 4.0 is due to the management of big data. For this, different online platforms such as Google App Engine, Amazon Web Services are available. Google App Engine is a scalable cloud hosting service providing a platform for developing and deploying web applications and can also be scaled as per requirements. Amazon Web Services is a cloud computing environment consisting remote computing services including processing, networking and storage resources on cloud. In this paper, effectiveness of blockchain has been showcased in the field of financial transactions.
\end{abstract}

\section{Keywords - Cryptography, blockchain, financial transactions, Credit Card}

\section{INTRODUCTION}

In the era of modernization where everything is getting digitized, everyone is concerned about security of e-money and digital wallets. Blockchain an emerging technology that supports and secures e-money in the form of bitcoin. In other words, the most common application of block chain is Bitcoin. In bitcoin users can transact funds directly with each other without the interruption of the middleman. Bitcoin is divisible, portable and irreversible. Bitcoin is more efficient and less costly [1]. The main advantage of using blockchain is the security be-cause of usage of hash values used in it.

Blockchain is defined as a collection of records linked to each other like a link list, each node of the list contains some hash value making it more secure. The hash values for each node is calculated based on hash values of previous block. Each node also contains the timestamp and the transaction date. This means that each current block contents depend upon all the previous blacks. To create each new block, a hash value is needed of its previous block, since each block depends upon the previous block, it becomes a chain of blocks, hence the name block chain. Thus, making it more secure and resistant. It works in a peer to peer net-work [2]. It can also be said that it is a kind of ledger technology which is distributed among the network and sends every digital transaction to all peers in a de-centralized network of blockchain nodes which verifies the received transaction. The whole network reached to an agreement regarding the correctness of

Received: September 21, 2019

Reviewed: November 18, 2019

Accepted: December 2, 2019

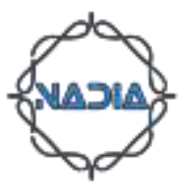


history of all the transactions or records automatically. This also helps in making the database of transaction as immutable and transparent.

Blockchain was first invented in the form of cryptocurrency by a group of people known as Satoshi Nakamoto in 2008. Cryptocurrency is referred to the digital currency which relies on cryptographic technology for the verification and securing the funds transfer and currency generation. They are generally based on the concept of money as information which is sent as a message in the form of strings of bits. The primitive form of block chain was Merkle tree (hash tree). Merkle tree was a chain of blocks containing data where the last block contained the his-tory of all the previous blocks. Every transaction is stored as a ledger in a block. Before a transaction is added to the chain, it is first verified by a distributed system. A Consensus needs to be established when majority of nodes or all nodes in the network are in possession of the valid block of the transactions. Block chains are considered more secure because they are not easily accessible for modifications and alterations. In fact, if a single change is required all the blocks from that first block to last block needs to be altered, which makes it secure [3].

Blockchain has been developed using three technologies: private and public key cryptography, internet and networking protocols. The advantage of using this combination of technologies is that during transactions and communications, no third party is required [4].

Blockchain in financial transactions also helps in avoiding the problem of double spending as it keeps an eye on the complete history of the transactions. Blockchain applications are not limited only to Bitcoin but there are various other applications for Blockchain which includes Smart contracts, Asset verification, Cryptocurrency vault, Crowdfunding, Insurance sector, Digital payment system, Internet of Things and many more. Blockchain technology is rapidly developing technology in both public and private organization.

Consensus network is a network where the blockchain is updated according to a consensus among the miners. The blockchain transaction is verified by implementing consensus with an algorithm that can be based on mainly two types of proofs: one is proof of work and another is knows as proof of stake. In proof of work, the participant of the blockchain network also known as miners compete among themselves to validate and adding the new block of valid transactions into the chain by solving a complex puzzle using their own computing resources. The miner who solves the puzzle first and succeed in adding new block to in chain are awarded with a small amount of commission or transaction fee. In Proof of concept consensus validation mechanism, the miners do not compete to solve the puzzle but they are chosen on the basis of how much stake one is having, hence making the chance of verifying a transaction directly related to his stake in the blockchain. The miner with a greater number of digital coins will get more computing power to validate and add the new transaction in the blockchain.

\section{MOTIVATION}

The Primary motivation for undertaking the current research work is to design and implement the effective and efficient BLAST Algorithm (see Algorithm 1,2,3) which have been formulated for the effective utilization of Blockchain Technology. To the best of our knowledge, no such algorithms exist in the literature. Also, with the rise of usage of cards in online payment, the need of secure transaction arises. This also includes the validation of the entered details and encrypting the same.

\section{LITERATURE SURVEY}

Blockchain is considered to be a decentralized system owing to the way it stores data. Data is not stored in a centralized storage system but in chain of blocks. So, in [5], Wright et al. have discussed this decentralized system, uses, ad-vantages and disadvantages and 
future impact on society along with the risks involved. Further, they have said that soon there will be a need for establishing rules and regulations, owing to its widespread popularity. Blockchain technology being a decentralized system and self-executing smart contracts administration, the laws may be termed as LexCryptographia. Some principles related to the adoption of legal rules and regulations have also been discussed.

The concept which makes crypto currency so important is the Blockchain. Basically, blockchain provides security to the transactions. Transparency and security are core areas among the financial transactions. Security is accomplished by hashing. Mining of Blockchain is also important. So, in [6], Daniel Kraft pro-posed a model for mining which helps in finding the average block times. The model is based on the time dependent intensity Poisson process. Secondly, to re-solve the network issues in block times, that is high block growth and exponentially rising hash rates, a Bitcoin based solution has been analyzed. The proposed improved method has better performance along with more stability in average block times over longer period of time. Also, it works well in constant as well as exponentially growing hash rates. The simulations have been carried out both on real time datasets and autocorrelation growth scenarios. In [7], according to Aggelos Kiayias et al., the process of mining is analogous to the concept of block chain. Each and everything is transparent but still it cannot be used by unauthorized users. So, in this work, they describe how the process of mining is identical to the process of Blockchain. Author(s) studies mining as a game. Two games have been considered. In first, miners inform immediately about the release of the mine after it has been mined but keep the secret which exact mine will be mined next. In second game, others miners are only informed that mining has been done but which mine has been mined is not informed to other miners. According to the results, best results matched the expected behavior when the computational power is small.

In [8], Gareth W. Peters et. al., have discussed the Blockchain technology and its related advancements. Further, applications of Blockchain technology have been listed, mainly focusing on the application in the field of banking. Author(s) have discussed in detail the working, benefits, issues and challenges in implementation of ledger based Blockchain technology in banking systems services. Digitization has changed the way one used to live in this world. "Smart" is the new trend in this world. Cities are transforming towards smart cities. So, in [9], Jianjun Sun et al. proposed a conceptual framework for the development of the smart cities with respect to shared economy. The proposed triangle framework is based on three dimensions: human, technology and organization. Smart cities involve smart technology. So, to accomplish those tasks new trending technology is used. So, it was described how Blockchain technology helps in the development of smart cities with respect to sharing economy.

In [10], Sarah Underwood explained the Blockchain technology. Main focus was on describing the benefits and the applications of Blockchain. Future scope has too been listed. In [11], Marko Vukolic has done comparative study between the traditional Blockchain technology based on the concept of proof of work (PoW) and the latest improvised version of Blockchain technology, such as Ethereum, having better performance and scalability. The newer Blockchain technology works on distributed computing. It is based on database replication protocols such as classical state machine replication. The state machine replication protocol has many variants. The Blockchain technology is based on Byzantine fault tolerant. Major area of discussion in this work is the comparison of scalability limits on both the models. Some solutions and some open challenges in overcoming the issues of scalability have also been listed.

In [12], Xu, Xiwei, et al., have compared two different scenarios for data storage. First is based on Blockchain technology where a decentralized storage architecture is maintained. Second is traditional centralized storage system. Another area explored by the author(s) is using Blockchain as a software connector, along with comparing it with existing software connectors. Blockchain architecture has also been explained in detail. 
Author(s) have presented their experience on Block-chain after implementing practical knowledge on different projects. The paper has been concluded by listing the trade-offs in the technology.

Blockchain has been widely adopted all over the world. So, in [13], Yli-Huumo, Jesse, et al. have conducted a study on analyzing the area of research, key challenges in the implementation of the technology and future scope of this technology.

In [14], Pass et al. have proposed an improvised version of Nakamoto's proto-col. According to Nakamoto's protocol, firstly, anyone can leave or join the execution of protocol. Secondly, the protocol execution is independent of the players identity. In the framework proposed by Pass et al., consistency and liveliness properties remain same as in Nakamoto's protocol but with better computational performance by providing enhanced probability parameters. The proposed proto-col is named fruit chain protocol. The fruit chain protocol reduces the mining pool need.

Different protocols are present in Blockchain. First Blockchain protocol is ouroborus which is a secured proof of stake. In Kiayias, Aggelos, et al. [15], Ouroborus protocol is com-parable to Bitcoin Blockchain protocol. Ouroborus protocol has been explained in detail in this work. A reward mechanism has been proposed based on the proof of stake. The proposed mechanism proves that actual behaviors are observed approximately to Nash equilibrium which neutralizes the attacks such as selfish mining.

In [16], Korpela et al. have studied the supply chain mechanism. Main focus had been on reducing the computation costs involved in the procedure. A cost-effective method based on cloud computing has been proposed. In [17], Michael et al. have explored the Blockchain technology and is applications, areas which can lead to issues is block chain concepts and government regulations in the implementation of Blockchain technology. This work also lists the various agencies related to Blockchain.

In Zheng, Zibin, et al. [18], a comprehensive literature survey has been conducted on the applications of the Blockchain. Blockchain architecture and the block structure has been explained in the detail. Along with it, Blockchain definition, different algorithms for Blockchain and the challenges in the implementation of the Blockchain ap-plications have been discussed. Solutions have also been posted for the challenges. Future perspectives for the Blockchain applications have also been proposed

In [19] Gupta, Shubham, et al. and [20] Garg, Neelansh et al. discuss about a new Cryptographic technique "Cyclic Cryptographic Technique" and Pentaplicative Cipher Technique respectively which has been compared with traditional Cryptographic technique(s) such as Caesar Cipher, Vigenere Cipher and Affine Cipher and the same techniques can be further enhanced by using the Blockchain technology. In [21] Jain, Ishu et al., the work is similar to the BLAST technique as they also perform simulation on the Credit Card details and design and develop CAVEAT: Credit Card Vulnerability Exhibition and Authentication Tool, the tool can further be strengthened by adopting the Blockchain technology.

\section{METHODOLOGY ADOPTED}

The objective of carrying out the current research work was to design and develop state of the art blockchain that contains the User Credit Card Information secured using SHA 256 Algorithm. All financial transactions need to be conduct-ed securely on the web as due to involvement of multiple information exchange and the risk of information getting exposed increases exponentially with every information exchange entity. Also, Blockchain is capable of recording every single detail of the transaction in an encrypted single digital register which substantially reduces the number of information exchange entities.

The research work also includes the validation of the card number by using in-put data as customer's credit card number along with three-digit CVV and date of expiry in the 
format of mmyyyy. The received credit card information is checked using LuhnMod10 Algorithm, which is a simple check sum formula used as first line of defense by many ecommerce sites and banks to validate the card number. It generates the check digits by using the card digits and helps in reducing the likelihood of occurrence of typing errors occurred during input. The proposed application was programmed in Java programming language using ECLIPSE IDE. The detailed working steps adopted are as follows: -

1) The first task involved designing a Java based financial transaction OLTP application. The application would be used by E-Commerce Vendor. The application received following inputs from user before sending the data for verification and validation on the secure payment gateway: -

a. Credit Card Number

b. Credit Card Verification Value Number

c. Expiry Date.

2) After receiving the User Credit Card Information, the credit Card number is verified using LuhnMod10 Algorithm as detailed in Algorithm 1.

3) Create a concatenated string that combines the received Credit Card Number, Credit Card Verification Value Number and Expiry Date.

4) If the Length of this string is less than 25 , it is to be rejected or else create five blocks of five digits each, as detailed in Algorithm 2.

4.1 Each block contains following information: -

a) Previous block hash Value

b) Data

c) Timestamp

d) Current block hash value

4.2 The Previous hash Value field of the first block always contains a Zero.

5) Calculate the Hash value of the Current block by invoking the SHA 256 Algorithm, as explained in Algorithm 3.

6) The Chain of the block is then stored in the ArrayList.

\section{CONTRIBUTION: BLAST ALGORITHMS}

The Primary contribution towards the current research work is designing of ef-ficient BLAST Algorithm (see Algorithm 1,2,3) which have been formulated for the effective utilization of Blockchain Technology.

Algorithm 1: Received the Credit Card Information

\section{Notation}

CCN : Credit Card Number

CVV : Credit Card Verification Value

ED : Expiry Date on Card

CS : Concatenated String

Trigger: User Inputs the Credit Card Number

1. Check if the Credit Card Number is Valid $\mathrm{X} \leftarrow\{$ LuhnModAlgo $(\mathrm{CCN})\}$

2. If( $X$ is False $)$ 


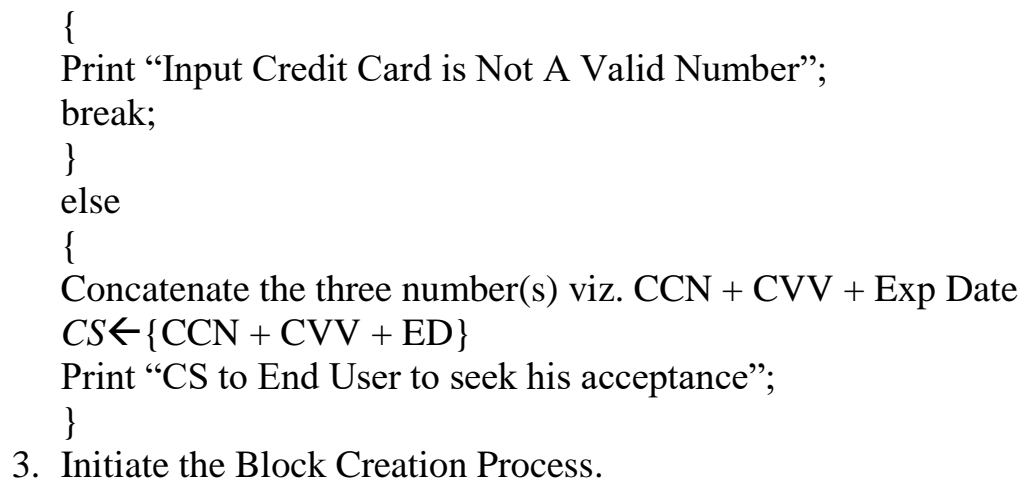

3. Initiate the Block Creation Process.

\section{Algorithm 2: Block Creation Process}

\section{Notation:}

CS : Concatenated String containing Credit Card Number

PH : Previous Hash Value

$\mathrm{CH}$ : Current Hash Value

DoB : Data for Block

Trigger: String containing Luhn Mod10 Algorithm Verified Credit Card Number is received

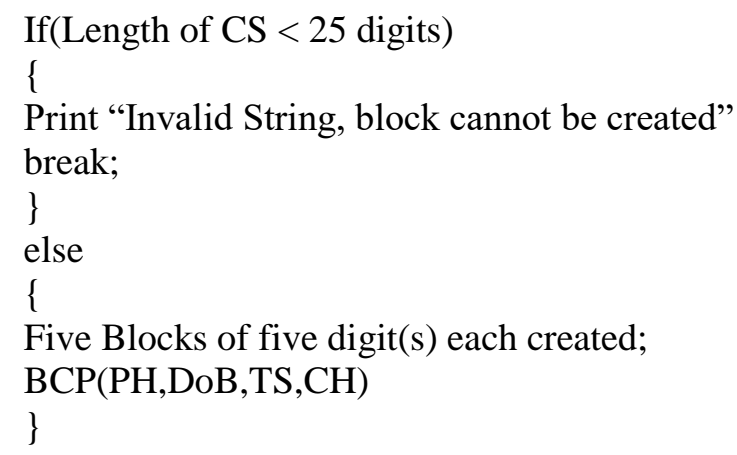

Algorithm 3: Hash Calculation

\section{Notation}

CS : Concatenated String containing Credit Card Number

PH : Previous Hash Value

$\mathrm{CH}$ : Current Hash Value

Trigger: Block Containing the User Credit Card Information received

1. Generate HashValue by Applying SHA256 Algorithm

2. $\mathrm{HC} \leftarrow[\mathrm{HC}(\mathrm{SHA} 256(\mathrm{PH}, \mathrm{DoB}, \mathrm{TS})]$

3. //PH for First Block is Zero and Next Block contains Hash of Previous Block

4. $\mathrm{BR} \leftarrow[\mathrm{PH}, \mathrm{DoB}, \mathrm{TS}, \mathrm{HC}]$

\section{EXPERIMENTAL SETUP}

Blockchain concepts were applied on transferring details in an encoded manner. Blockchain implementations have been conducted on Eclipse IDE using JAVA Programming language. 


\section{RESULT ANALYSIS}

For simulation, card details were provided to the application which then checks the details using Luhn's mod 10 algorithm and sends an error message if the data was not correct. Once the data is verified, the block chain blocks are generated and encrypted using SHA 256 algorithm. Figure 1 displays the output on the Eclipse console screen.

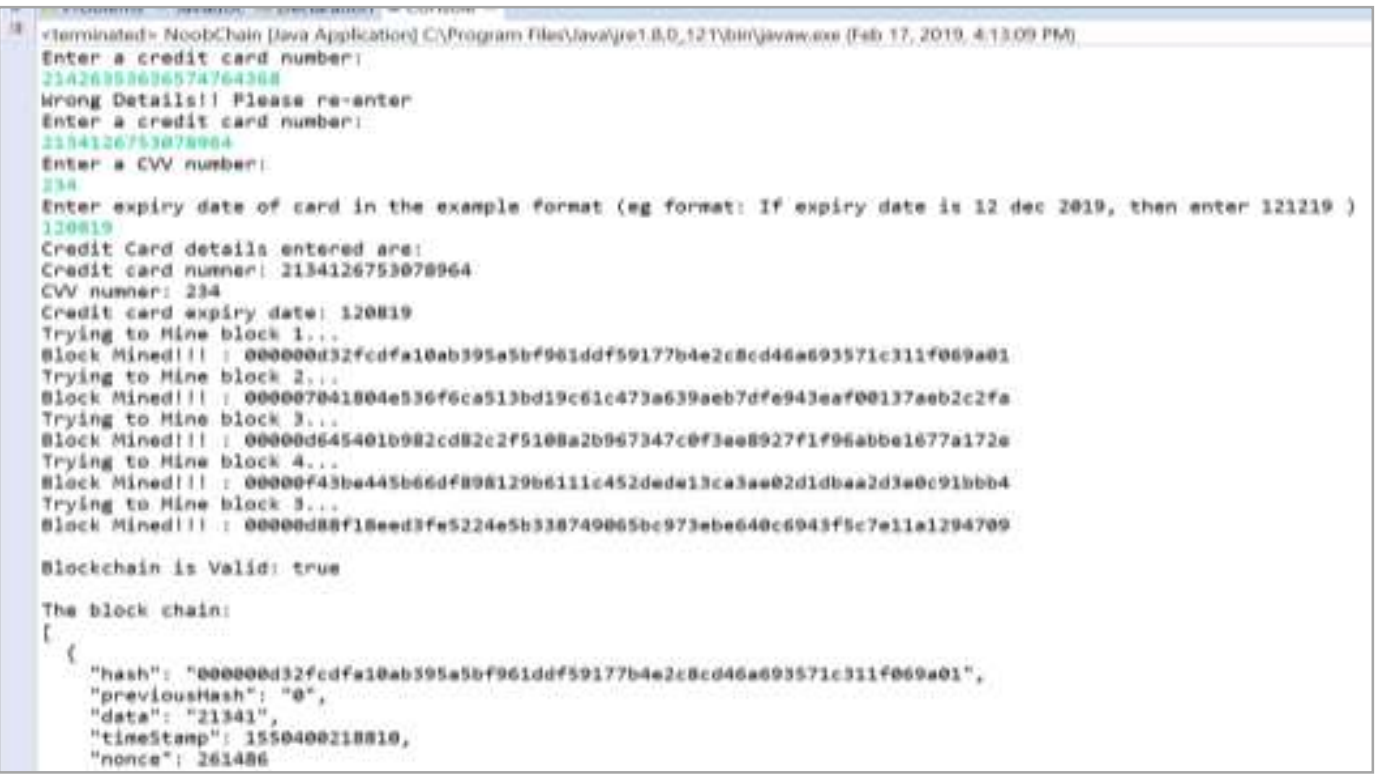

Fig. 1 Sample Snapshot 1 of BlockChain - Credit Card Application designed using JAVA ECLIPSE IDE.

\section{CONCLUSION}

Blockchain is an emerging technology and will continue to flourish in future due to the ingenious way it solves for a transparent, distributed consensus network that is resistant to manipulation or takeover by a central authority. Nowadays, businesses are moving towards blockchain technology. In this work, introduction to blockchain has been taken into consideration. To show the effectiveness of blockchain technology, blockchain has been used in the financial transactions to make them secure. The credentials of the user need to be sent via secured medium to minimize the risk of hacking of information after proper validation of the card information.

\section{REFERENCES}

[1] “Learning Portal.” Bitcoin For Beginners. https://www.blockchain.com/learning-portal/bitcoin-faq.

[2] "Blockchain." Wikipedia. Wikimedia Foundation. https://en.wikipedia.org/wiki/Blockchain

[3] LafailleChantelle, Chantelle. "What Is Blockchain Technology? An Easy Guide For Beginners (2019)." Invest In Blockchain, April 8, 2019. https://www.investinblockchain.com/what-is-blockchaintechnology.

[4] "Blockchain 101." CoinDesk. https://www.coindesk.com/information/what-is-blockchain-technology.

[5] Kraft, Daniel. "Difficulty control for blockchain-based consensus systems." Peer-to-Peer Networking and Applications 9.2 (2016): 397-413.

[6] Kiayias Aggelos, Koutsoupias Elias, Kyropoulou Maria, Tselekounis Yiannis. "Blockchain mining games." Proceedings of the 2016 ACM Conference on Economics and Computation. ACM, 2016.

[7] Peters, Gareth W., and Efstathios Panayi. "Understanding modern banking ledgers through blockchain technologies: Future of transaction processing and smart contracts on the internet of money." Banking beyond banks and money. Springer, Cham, 2016. 239-278.

[8] Sun, Jianjun, Jiaqi Yan, and Kem ZK Zhang. "Blockchain-based sharing services: What blockchain technology can contribute to smart cities." Financial Innovation 2.1 (2016): 26.

[9] Underwood, Sarah. "Blockchain beyond bitcoin." Communications of the ACM 59.11 (2016): 15-17. 
[10] Vukolić, Marko. "The quest for scalable blockchain fabric: Proof-of-work vs. BFT replication." International workshop on open problems in network security. Springer, Cham, 2015.

[11] Xu, Xiwei \& Pautasso, Cesare \& Zhu, Liming \& Gramoli, Vincent \& Ponomarev, Alexander \& Tran, An Binh \& Chen, Shiping. "The blockchain as a software connector." 2016 13th Working IEEE/IFIP Conference on Software Architecture (WICSA). IEEE, 2016.

[12] Yli-Huumo, Jesse, Deokyoon, Sujin, Sooyong, Kari. "Where is current research on blockchain technology? — a systematic review." PloS one 11.10 (2016): e0163477.

[13] Pass, Rafael, and Elaine Shi. "Fruitchains: A fair blockchain." Proceedings of the ACM Symposium on Principles of Distributed Computing. ACM, 2017.

[14] Kiayias Aggelos, Russell Alexander, David Bernardo, Oliynykov Roman. "Ouroboros: A provably secure proof-of-stake blockchain protocol." Annual International Cryptology Conference. Springer, Cham, 2017.

[15] Korpela, Kari, Jukka Hallikas, and Tomi Dahlberg. "Digital supply chain transformation toward block chain integration." proceedings of the 50th Hawaii international conference on system sciences. 2017.

[16] Michael, JW, Alan Cohn, and Jared R. Butcher. "Blockchain Technology." The Journal (2018).

[17] Zheng Peilin, Zheng Zibin, Luo Xiapu, Chen Xiangping, Liu Xuanzhe. Blockchain challenges and opportunities: a survey." International Journal of Web and Grid Services 14.4 (2018): 352-375.

[18] Gupta, Shubham, Rahul Johari, Prachi Garg, Kalpana Gupta. "C 3 T: Cloud based Cyclic Cryptographic Technique and it's comparative analysis with classical cipher techniques." 2018 5th International Conference on Signal Processing and Integrated Networks (SPIN). IEEE, 2018.

[19] Garg, Neelansh, Harshit Bhatia, and Rahul Johari. "Pentaplicative Cipher Technique." International Conference on Innovative Computing and Communications. Springer, Singapore, 2019.

[20] Jain, Ishu, Rahul Johari, and R. L. Ujjwal. "CAVEAT: credit card vulnerability exhibition and authentication tool." International Symposium on Security in Computing and Communication. Springer, Berlin, Heidelberg, 2014. 